\title{
Morphological quantification of proliferation-to-invasion transition in tumor spheroids
}

Yi He ${ }^{\mathrm{a}}$, Ling Xiong ${ }^{\mathrm{a}}$, Xuejuan Gao ${ }^{\mathrm{b}}$, Miaomiao Hai ${ }^{\mathrm{a}}$, Yanping Liu ${ }^{\mathrm{a}}$, Gao Wang ${ }^{\mathrm{a}}$, Guo Chen ${ }^{\mathrm{a}}$, Jianwei

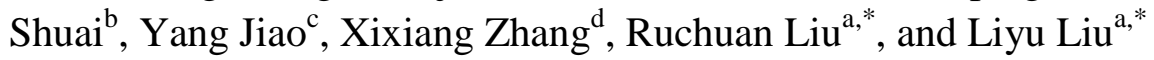

${ }^{a}$ Chongqing Key Laboratory of Soft Condensed Matter Physics and Smart Materials, College of Physics, Chongqing University, Chongqing, 401331, China

${ }^{\mathrm{b}}$ Department of Physics, Xiamen University, Xiamen, 361005, China

${ }^{\mathrm{c}}$ Materials Science and Engineering, Arizona State University, Tempe, AZ 85287, USA

${ }^{\mathrm{d}}$ Division of Physical Science and Engineering, King Abdullah University of Science and Technology, Thuwal, 23955-6900, Saudi Arabia

*Corresponding author.

Keywords: Living-cell device; Tumor spheroids model; Cancer invasion; Image processing; Quantitative analysis; Transition time

\section{Abstract}

Background: Metastasis determines the lethality of cancer. In most clinical cases, patients are able to live with tumor proliferation before metastasis. Thus, the transition from tumor proliferation to metastasis/invasion is essential. However, the mechanism is still unclear and especially, the proliferation-to-metastasis/invasion transition point has not been well defined. Therefore, quantitative characterization of this transition is urgently needed.

Methods: We have successfully developed a home-built living-cell incubation system combined with an inverted optical microscope, and a systematic, quantitative approach to describing the major characteristic morphological parameters for the identification of the critical transition points for tumor-cell spheroids in a collagen fiber scaffold.

Results: The system focuses on in vitro tumor modeling, e.g. the development of tumor-cell spheroids in a collagen fiber scaffold and the monitoring of cell transition from proliferation to invasion. By applying this approach to multiple tumor spheroid models, such as U87 (glioma tumor), H1299 (lung cancer), and MDA-MB-231 (breast cancer) cells, we have obtained quantitative morphological references to evaluate the proliferation-to-invasion transition time, as well as differentiating the invasion potential of tumor cells upon environmental changes, i.e. drug

\footnotetext{
*Email: lyliu@cqu.edu.cn

*Email: phyliurc@cqu.edu.cn
} 
application.

Conclusions: Our quantitative approach provides a feasible clarification for the proliferation-to-invasion transition of in vitro tumor models (spheroids). Moreover, the transition time is a useful reference for the invasive potential of tumor cells.

General significance: This quantitative approach is potentially applicable to primary tumor cells, and thus has potential applications in the fields of cancer metastasis investigations and clinical diagnostics.

\section{Introduction}

Metastasis is the primary cause of malignant cancers, which can result in the death of cancer patients[1, 2]. Among the multiple processes involved in metastasis, tumor-cell invasion is the crucial step. Previous work demonstrated that tumor cells can invade in a three-dimensional (3-D) environment via different ways, i.e. as single cells[1, 3, 4], or as collective forms (i.e. protrusions, cell sheets)[5-11]. Collective cell invasion has been observed in pathological samples of oral squamous carcinomas, ductal breast carcinomas, and rhabdomyosarcoma[10, 12]. To reveal their mechanisms, numerous methods have been developed in laboratory, including theoretical models of morphology[13, 14], interfacial geometry[15], morphology of cells[16], lab-on-a-chip microfluidic devices[17], ultrasound[18], Raman technique[19], image analysis[20-22], etc. Traditionally, the Transwell assay has been a widely commercialized technique for evaluating tumor-cell invasiveness[23, 24], although it still has limitations due to its non-three-dimensional microenvironment. In contrast, multicellular tumor spheroids (MCTS) are the most used as in vitro 3-D models for collective cancer metastasis[25-27]. In these studies, the collective invasions of spheroids have been extensively investigated. To date, the majority of studies of cancer-cell invasion have focused on the proliferation processes or post-invasion processes and the mechanisms at either stage[28-31]. However, study on the transition, i.e. how tumor proliferation evolves into collective invasion is still lacking. Nevertheless, the proliferation-to-invasion (PTI) transition is an essential step, but is insufficiently defined, likely because of technical limitations. In addition, this transition point could also be critical in the evaluation of the potential for tumor-cell invasion, and thus its quantitative characterization is urgently needed. In this decade, for the detection of cell migration or invasion in vitro, the IncuCyte ${ }^{\mathrm{TM}}$ (Essen BioScience) chemotaxis system is one of a commercially devices, which is a high through-put integrated system for real-time visualization and automated analysis of chemotactic cell migration/invasion within a tissue culture incubator. Although these 
systems are sophisticated, the high cost of setting up these kinds of instruments is one of the main disadvantages[32]. Whereas, we customized a home-built living-cell incubation system, developed an automated and quantitative approach to provide a feasible clarification for the PTI transition of in vitro tumor spheroid models.

\section{Materials and methods}

\subsection{Cell culture and extracellular matrix preparation}

U87 (glioma tumor) cells were obtained from the Institute of Biochemistry and Cell Biology, Chinese Academy of Science (Shanghai, China), H1299 (lung cancer) cells were obtained from the Cell Bank of the Chinese Academy of Sciences (Shanghai, China), MDA-MB-231 cells were obtained from China Infrastructure of Cell Line Resources (Beijing, China). All U87 cells, H1299 cells and MDA-MB-231cells were labeled with green fluorescent protein (GFP) and GFP were transfected into cells of three cell lines by lentivirus infection method. Culture media were DMEM/F12 medium (10-092-CVR, Corning, USA) for U87 cells, RPMI 1640 medium (10-040-CVR, Corning, USA) for H1299 cells, DMEM medium (10-013-CVR, Corning, USA) for MDA-MB-231 cells. In all media, 10\% fetal bovine serum (FBS, 35-010-CV, Corning, USA) and 1\% penicillin/streptomycin (P/S, 30-002-CI, Corning, USA) were supplemented. All cells were cultured in an incubator (C150, Binder, Germany) at $37.0{ }^{\circ} \mathrm{C}$ with $5.0 \% \mathrm{CO}_{2}$.

According to the manufacturer's instructions, Neutralized collagen I solutions were prepared by mixing buffer solutions of sterile deionized water, 10X PBS (70013-032, Gibco, USA) and 1 mol/L $\mathrm{NaOH}$ (795429, Sigma-Aldrich, USA) with the high concentration collagen I (354249, Corning, USA) on ice-pack. The final collagen I concentration was $2.0 \mathrm{mg} / \mathrm{mL}$ for all the tests.

\subsection{Spheroids formation}

In this study, we present a systematic in vitro approach (Fig. 1) to analyzing cell invasiveness quantitatively utilizing MCTS as 3-D models. In this approach, we combine the MCTS technique with a home-built living-cell incubator on top of a microscope to track the changes in morphology of spheroids. From time snapshots of each spheroid, we analyzed the images and designed a set of morphological aspects to define the critical time of PTI transition (Fig. 1(a)) for MCTS. Spheroids were formed by a method similar to that reported by Ivascu et al[33]. During spheroid formation, the incubation media were same as the corresponding culture media above, except $3.5 \%$ Matrigel (356237, Corning, USA) was also added to the culture medium for MDA-MB-231 cells. This transition time could be an excellent reference for evaluating the potential of tumor-cell invasion. To demonstrate this approach, we used GFP-labeled U87 cells, H1299 cells, and MDA-MB-231 cells to 
evaluate the PTI transition time and its change upon drug implementation. As displayed in Fig. 1(b), in a 96-well round bottom ultra-low attachment (ULA) plate (7007, Corning, USA), the tumor cells $\left(1.0 \times 10^{4}\right.$ cells $\left./ \mathrm{mL}\right)$ were incubated for 96 hours to form a stable spheroid in each well (Fig. S1). Subsequently, the spheroids were transferred to another 96-well flat bottom ULA plate (3474, Corning, USA) to monitor tumor spheroid invasion; the culture medium with $2.0 \mathrm{mg} / \mathrm{mL}$ collagen I was expected to simulate a 3-D microenvironment in vitro for spheroids[34-36].

\subsection{Fabrication of home-built living-cell device}

A home-built incubator was then used to maintain a stable and uniform temperature, $\mathrm{CO}_{2}$ concentration, and humidity for the spheroid culture on top of optical microscope. As illustrated in Figs. 1(c) and 1(d), the customized living-cell system consisted of a controller and a sealed chamber to hold the plate. A water groove maintained the humidity of incubator inside to reduce evaporation of culture medium. Four heaters and four temperature sensors located in the chamber stage, near the spheroids, the chamber cap, the water bath, and the microscope objective, maintained a uniform temperature of $37.0^{\circ} \mathrm{C}$. This design enabled a stable culture temperature and humidity around spheroids in the chamber to be maintained, which was essential for extended live imaging during the entire PTI transition process. There was also a gas inlet admitting $5.0 \% \mathrm{CO}_{2}$, as monitored by the controller, to maintain a $\mathrm{pH}$ of $\sim 7.4$ in the medium.

\subsection{Image processing}

The spheroid was imaged by a CCD camera (Neo 5.5 sCMOS, Andor, USA), the imaging rate was 10 min per frame for U87 spheroids. The light intensity was $130 \mathrm{~mW}$ and the exposure time was set at $200 \mathrm{~ms}$. Figure 2(a) illustrates the procedure for image processing. First, each fluorescent image was transformed into a grayscale image. Next, all fluorescence noises were reduced using the built-in functions of MATLAB software (MathWorks, USA). Then, Otsu's method[37] was applied to automatically transform the smoothed grayscale image into a binary one. Subsequently, all holes inside were filled, and the largest connected component was identified as the spheroid. Finally, the centroid of spheroid and the distance of boundary to the center were calculated for further analysis. The overlap of boundaries at $0,40,80$, and $120 \mathrm{~min}$ of the same sample are displayed in Fig. 2(b) (see Fig. S2 for all images).

\subsection{Boundary smoothing}

Further processing of the raw boundary data is described in Fig. 3. Since the raw boundary data (Fig. 3(a1)) are not uniform in size across time frames and spheroids, a unified polar coordinate system is more appropriate for reformatting the boundary. Using an image resolution of $2560 \times 2160$ pixels, the circumference of the spheroid edge was divided equally into 300, 600 or 720 portions, and no distinct difference was found in following PTI identification. Thus, all following analysis used 
300 portions for division. In most of divided parts (Fig. 3(a2)), there are multiple boundary points connected to each other in a complicated manner. To eliminate this complexity, while still catching the protrusion characteristics of collective cell invasion, the maximum distance $(d)$ of data points from centroid was used as the representative radial coordinate for each divided part, as displayed in Fig. 3(a2-a3). Finally, smoothed boundary was presented in Fig. 3(a4).

\subsection{Boundary identification}

The smoothed and well-formatted boundary (Fig. 3(a4)) was further transferred to the frequency domain using discrete Fourier transform (DFT) in MATLAB. In this domain, the frequency describes the sharpness of boundary variations. The collective tumor cell invasion leads to sharp protrusions from spheroids, in contrast to the relatively smooth boundary during tumor-cell proliferation. Therefore, the high-frequency portion of DFT trajectory contains the morphology changes due to invasion, while the low-frequency part is "baseline". Empirically, the separation frequency (Fig. 3(b)) is 15 , and the corresponding low-frequency portion displays the major feature of smooth boundary of proliferation (see Fig. S4 for details). It can be seen from Fig. 3(c) that frequency filtration of boundary data $\left(d_{h f}\right)$ successfully catches the sharp protrusions by removing the large, but smooth, features of proliferation morphology.

\section{Results and discussion}

Significant morphological changes are usually the sign of a transition from proliferation to invasion for "invasion-competent" tumor spheroids. In contrast, "invasion-incompetent" spheroids (i.e. MCF-10A) undergo only some non-invasive expansive growth in collagen environment[28]. In previous work, morphological parameters were utilized to quantify a spheroid invasion feature, such as geometric parameters (spheroid area, perimeter, radius, and volume)[7, 21, 30, 38-40], isolation of core and invasive compartments[7, 21, 38, 41], surface roughness or irregularity[7, 39, 42, 43]. These methods have provided useful references to this study. To identify the PTI transition, morphological features, i.e. area and boundary variations, are useful for this purpose. First, invasion protrusions of spheroids are expected to result in larger increments to the perimeters of their 2-D images than the enclosed area[42]. In contrast, the proliferation of spheroids increases both perimeter and area comparably. Therefore, we define a dimensionless perimeter ${ }^{2}$-to-area ratio $\left(r_{p t a}\right)$ to quantify the irregular shape of spheroids as a result of collective cell invasion. The sharp change appearing in the first derivative of $r_{p t a}$ was defined as transition time. The $r_{p t a}$ for a U87 spheroid (Fig. 3(d), black curve) clearly displays a large increment between 40 and 50 min. This suggests a PTI transition time $\left(\tau_{p t i}\right)$ of $40 \mathrm{~min}$ for this spheroid. Furthermore, the invasion protrusions also result in an increase in the roughness of the spheroid circumference, which could be characterized by the standard deviation[43], $d_{s t d}$, of the relative boundary-to-center distances (i.e. $d_{h f} / d_{\text {avg }}$ ). The result in Fig. 3(d) 
(red curve) gives a similar $\tau_{p t i}$ of $40 \mathrm{~min}$ as transition time where the sharp change appeared in the first derivative of $d_{s t d}$.

Due to parameter $r_{p t a}$ and parameter $d_{s t d}$ not well determine their mathematical transition points, the parameters describing protrusions characteristics of tumor-cell invasion of spheroids are considered. The protrusions exhibit various outreach heights and shapes, i.e. the protrusion height (h)[7] and the height-to-width ratio $\left(r_{h t w}\right)$ [11]. While there are many protrusions, only a limited number of them are invasion-related. This is because invasive protrusions are expected to be rare events at the PTI transition, i.e. the starting point of collective cell invasion. Taking all protrusions of a spheroid at each time snapshot into account, the statistics of $h$ and $r_{h t w}$ (see definitions in Fig. S5) gives the two-dimensional probability distribution (Fig. 4(a)) for the aforementioned U87 spheroid. The majority of protrusions (i.e. with small $h$ and low $r_{h t w}$ ) display a dense, large domain at the bottom-left corner of the histogram. Such protrusions exist even before the start of invasion, and thus are due to the proliferation dynamics of tumor spheroids. In contrast, the upper-right corner of the histogram (yellow square in Fig. 4(a)) includes domains of limited probability, corresponding to protrusions with both large $h$ and high $r_{h t w}$. Since the proliferation of tumor spheroids could hardly result in these protrusions, they are excellent candidates for the identification of invasive protrusions. Accordingly, in the following two-parameter justifications, the thresholds of $h(18 \mu \mathrm{m})$ and $r_{h t w}(0.55)$ are selected to include the majority of upper-right corner domains while excluding interference from the intense bottom-left corner domains (i.e. peak probability > 0.003).

Although two-parameter justification gives excellent results for identifying invasive protrusions, finding the correct two-parameter thresholds is more complicated. To simplify parameter selection, single-parameter justifications are also applied. Here, each threshold has to be higher to avoid the intense probability domains (i.e. peak probability > 0.003) next to the bottom-left corner, i.e. $20 \mu \mathrm{m}$ for $h$ and 0.6 for $r_{h t w}$ (see SI Sec. VII for details of parameter thresholds for all spheroids in the experiments). Nevertheless, single-parameter justifications also select rare protrusion events and are easier to use, but the selection of protrusions is slightly different than that by means of two-parameter justification.

With these thresholds for $h$ and $r_{h t w}$ in hand, the number of protrusions related to collective tumor cell invasion were counted for each spheroid per time snapshot. Figure 4(b) displays the trajectories of occurrences over time. It is clear that the first protrusions of this U87 spheroid with the aforementioned characteristics appear between 40 and $60 \mathrm{~min}$. This suggests the $\tau_{p t i}$ to be $40 \mathrm{~min}$ (from both two-parameter justification and $r_{h t w}$ value) and $50 \mathrm{~min}$ (from $h$ value), consistent with that from the $r_{p t a}$ and $d_{s t d}$ measurements (Fig. 3(d)). This consistency suggests the success of the thresholds for $h$ and $r_{h t w}$, as defined in Fig. 4(a). Therefore, large value of $h$ and high value of $r_{h t w}$ are the morphological characteristics of invasion protrusions from tumor spheroids. These features 
provide an experimental method for identifying the invasiveness of spheroids for further investigation, and in our opinion, the two-parameter justification gives the best selection.

The above approaches are suitable for application to various types of tumor cells and have also been applied to MDA-MB-231 and H1299 cells. In all cases, the values of $\tau_{p t i}$ measured by morphological parameters $r_{p t a}, d_{s t d}, h, r_{h t w}$, and the combination of $h$ and $r_{h t w}$ from the series of fluorescence images give consistent results for each cell line (see details in Table 1), where the variations in $\tau_{p t i}$ among spheroids are within the experimental time resolution. Such variations are due to spheroid variations and, inadvertently, differences in their culture conditions. Nevertheless, the average of multiple approaches gives a better measurement of $\tau_{p t i}$ than the single-parameter justifications, by avoiding accidental measurement errors. All experiments were at least repeated three times, the $\tau_{p t i}$ from these measurements $(n=3)$ are summarized in Fig. 4(c).

For further validation, we have measured the change in $\tau_{p t i}$ of tumor spheroids upon drug application. Discoidin domain receptor 1 (DDR1) is a receptor-tyrosine kinase widely expressed in many cancers[44-46]. Overexpression of DDR1 has been demonstrated to promote invasion and migration of tumor cells[47, 48]. In contrast, DDR1 inhibitors are expected to depress tumor-cell invasion. 7rh (SML1832, Sigma-Aldrich, USA) is a DDR1 inhibitor and has been proved to decrease the invasion velocity of gastric carcinoma cells[45]. It inhibits nasopharyngeal carcinoma cell proliferation and colony formation in a dose--dependent manner in vitro[49]. Treatment with 7rh at 5.0, 10.0, or $20.0 \mu \mathrm{M}$ inhibited cell-matrix adhesion within 2 hours[50]. When 7rh $(8 \mu \mathrm{M})$ was applied to U87, H1299 and MDA-MB231 spheroids, a slowdown in the PTI transition was observed. Figure 4(c) displays the results without $7 \mathrm{rh}$ and with $7 \mathrm{rh}$ treatment, and the change in $\tau_{p t i}$ is significant $(* p<0.05)$.

For the $\tau_{p t i}$ measurements, bright-field images could also be used instead of fluorescence images. For H1299 spheroids, both fluorescence and bright-field images have been recorded during experiments. The transition time measured (Fig. 4(c), gray and white columns for H1299 spheroids) is consistent with each other. However, since image processing for bright-field images to distinguish the spheroid boundary and background is more difficult than that for fluorescence images, spheroid boundary was extracted manually. Further improvements to bright-field image processing are underway, potentially for future applications to patient primary tumor cells, which are non-fluorescent. Additionally, this approach suggests that cell collective invasion is highly possible to invade into ECM as protrusions. On the other hand, in case of a sheet-like manner invasion, which might not be well coped with our quantification approach, further development may be needed.

\section{Conclusions}

In summary, a home-built living cell incubator was designed and constructed on top of an 
optical microscope to provide a uniform temperature and a stable culture environment for extended tracking of the PTI transition of tumor spheroids. Via 3-D in vitro models, spheroids of glioma tumor, lung cancer, and breast cancer cells were obtained and transferred into a medium of collagen I gel, where the PTI transition took place. Using serial images of these spheroids, a systematic, morphology-based quantitative approach was developed and utilized to identify the transition time. We used morphology parameters: $r_{p t a}, d_{s t d}, h, r_{h t w}$, the combination of $h$ and $r_{h t w}$, to characterize the PTI transition and obtain consistent values of $\tau_{p t i}$ for each cell line. We believe two-parameter ( $h$ and $r_{h t w}$ ) justifications to be the best for identifying the beginning stage of invasive protrusions, while other parameter justifications could help to ensure the precise measurement of $\tau_{p t i}$. In contrast, changes of parameter $r_{p t a}$ and $d_{s t d}$ are not sharp during PIT transition, hence parameter $h, r_{h t w}$, the combination of $h$ and $r_{h t w}$ are better in this manner. The timely identification of invasion protrusions could also assist further investigations of collective invasion mechanism, the suppression of U87, H1299 and MDA-MB-231 cells invasion by DDR1 inhibitor 7rh have been readily quantified as an increment of $\tau_{p t i}$. As the bright-field images may also be applicable, this approach has potential applications for primary tumor cell investigations, and could become a highly practical technique for rapid clinical diagnosis.

\section{Acknowledgments}

This work was supported by the National Natural Science Foundation of China (Grant Nos. 11674043 and 11604030), the Fundamental Research Funds for the Central Universities (2018CDJDWL0011), and the Fundamental and Advanced Research Program of Chongqing (Grant \#cstc2018jcyjAX0338), China.

\section{Appendix A. Supplementary data}

See the supplementary material for further details of U87, H1299, and MDA-MB-231 spheroids.

\section{References}

[1] W.J. Han, S.H. Chen, W. Yuan, Q.H. Fan, J.X. Tian, X.C. Wang, L.Q. Chen, X.X. Zhang, W.L. Wei, R.C. Liu, J.L. Qu, Y. Jiao, R.H. Austin, L.Y. Liu, Oriented collagen fibers direct tumor cell intravasation, Proc. Natl. Acad. Sci. U.S.A., 113 (2016) 11208-11213.

[2] R.S. Herbst, D. Morgensztern, C. Boshoff, The biology and management of non-small cell lung cancer, Nature, 553 (2018) 446-454.

[3] C. Veelken, G.J. Bakker, D. Drell, P. Friedl, Single cell-based automated quantification of therapy responses of invasive cancer spheroids in organotypic 3D culture, Methods, 128 (2017) 139-149. 
[4] P.C. Zhang, X. Han, J. Yao, N. Shao, K. Zhang, Y.F. Zhou, Y.L. Zu, B. Wang, L.D. Qin, High-Throughput Isolation of Cell Protrusions with Single-Cell Precision for Profiling Subcellular Gene Expression, Angew Chem Int Edit, (2019).

[5] P. Friedl, Y. Hegerfeldt, M. Tusch, Collective cell migration in morphogenesis and cancer, Int. J. Dev. Biol., 48 (2004) 441-449.

[6] G. Jacquemet, H. Hamidi, J. Ivaska, Filopodia in cell adhesion, 3D migration and cancer cell invasion, Curr. Opin. Cell Biol., 36 (2015) 23-31.

[7] C. Moriconi, V. Palmieri, R. Di Santo, G. Tornillo, M. Papi, G. Pilkington, M. De Spirito, M. Gumbleton, INSIDIA: A FIJI Macro Delivering High-Throughput and High-Content Spheroid Invasion Analysis, Biotechnol J, 12 (2017).

[8] A.G. Clark, D.M. Vignjevic, Modes of cancer cell invasion and the role of the microenvironment, Curr. Opin. Cell Biol., 36 (2015) 13-22.

[9] P. Friedl, S. Alexander, Cancer Invasion and the Microenvironment: Plasticity and Reciprocity, Cell, 147 (2011) 992-1009.

[10] F. Peter, L. Joseph, S. Erik, J.E. Segall, Classifying collective cancer cell invasion, Nat. Cell Biol., 14 (2012) $777-783$.

[11] R.C. Liu, K.N. Song, Z.J. Hu, W.B. Cao, J.W. Shuai, S.H. Chen, H. Nan, Y. Zheng, X.F. Jiang, H.F. Zhang, W.J. Han, Y. Liao, J.L. Qu, Y. Jiao, L.Y. Liu, Diversity of collective migration patterns of invasive breast cancer cells emerging during microtrack invasion, Phys. Rev. E, 99 (2019).

[12] J.J. Christiansen, A.K. Rajasekaran, Reassessing epithelial to mesenchymal transition as a prerequisite for carcinoma invasion and metastasis, Cancer Res., 66 (2006) 8319-8326.

[13] Y. Jiao, S. Torquato, Diversity of dynamics and morphologies of invasive solid tumors, AIP Adv., 2 (2012) 882.

[14] Y. Jiao, S. Torquato, Evolution and morphology of microenvironment-enhanced malignancy of three-dimensional invasive solid tumors, Phys. Rev. E, 87 (2013).

[15] J. Lee, A.A. Abdeen, K.L. Wycislo, T.M. Fan, K.A. Kilian, Interfacial geometry dictates cancer cell tumorigenicity, Nat Mater, 15 (2016) 856-862.

[16] M. Lekka, J. Pabijan, B. Orzechowska, Morphological and mechanical stability of bladder cancer cells in response to substrate rigidity, Biochim. Biophys. Acta-Gen. Subj., 1863 (2019) 1006-1014.

[17] Q.H. Fan, R.C. Liu, Y. Jiao, C.X. Tian, J.D. Farrell, W.W. Diao, X.C. Wang, F.R. Zhang, W. Yuan, H.B. Han, J.F. Chen, Y. Yang, X.X. Zhang, F.F. Ye, M. Li, Z.C. Ouyang, L.Y. Liu, A novel 3-D bio-microfluidic system mimicking in vivo heterogeneous tumour microstructures reveals complex tumour-stroma interactions, Lab Chip, 17 (2017) 2852-2860.

[18] D. Melodelima, D. Cathignol, Cancer treatment by ultrasound: Increasing the depth of necrosis, Appl. Phys. Lett., 84 
(2004) 5365-5367.

[19] S. Takanezawa, S.I. Morita, A. Maruyama, T.N. Murakami, N. Kawashima, H. Endo, K. Iijima, T. Asakura, T. Shimosegawa, H. Sato, Two-Dimensional Raman Correlation Analysis of Diseased Esophagus in a Rat, Appl. Phys. Express, 3 (2010).

[20] S. Dilipkumar, S. Montalescot, P.P. Mondal, Image reconstruction enables high resolution imaging at large penetration depths in fluorescence microscopy, Appl. Phys. Lett., 103 (2013).

[21] S. Blacher, C. Erpicum, B. Lenoir, J. Paupert, G. Moraes, S. Ormenese, E. Bullinger, A. Noel, Cell invasion in the spheroid sprouting assay: a spatial organisation analysis adaptable to cell behaviour, Plos One, 9 (2014).

[22] P. Choudhry, High-Throughput Method for Automated Colony and Cell Counting by Digital Image Analysis Based on Edge Detection, Plos One, 11 (2016).

[23] E. Primiceri, M.S. Chiriacò, F. Dioguardi, A.G. Monteduro, E. D'Amone, R. Rinaldi, G. Giannelli, G. Maruccio, Automatic transwell assay by an EIS cell chip to monitor cell migration, Lab Chip, 11 (2011) 4081-4086.

[24] K. Hatherell, P.-O. Couraud, I.A. Romero, B. Weksler, G.J. Pilkington, Development of a three-dimensional, all-human in vitro model of the blood-brain barrier using mono-, co-, and tri-cultivation Transwell models, J. Neurosci. Methods, 199 (2011) 223-229.

[25] E. Khain, L.M. Sander, Dynamics and pattern formation in invasive tumor growth, Phys. Rev. Lett., 96 (2006) 188103.

[26] K. Froehlich, J.D. Haeger, J. Heger, J. Pastuschek, S.M. Photini, Y. Yan, A. Lupp, C. Pfarrer, R. Mrowka, E. Schleußner, Generation of multicellular breast cancer tumor spheroids: comparison of different protocols, J. Mammary Gland Biol. Neoplasia, 21 (2016) 1-10.

[27] K. Jumpei, E. Hiroko, O. Hiroaki, I. Osamu, I. Hiroyasu, T. Masahiko, O. Masayuki, I. Masahiro, Retaining cell-cell contact enables preparation and culture of spheroids composed of pure primary cancer cells from colorectal cancer, Proc. Natl. Acad. Sci. U.S.A., 108 (2011) 6235-6240.

[28] S.P. Carey, A. Starchenko, A.L. McGregor, C.A. Reinhart-King, Leading malignant cells initiate collective epithelial cell invasion in a three-dimensional heterotypic tumor spheroid model, Clin. Exp. Metastasis, 30 (2013) 615-630.

[29] G. Jarockyte, D. Dapkute, V. Karabanovas, J.V. Daugmaudis, F. Ivanauskas, R. Rotomskis, 3D cellular spheroids as tools for understanding carboxylated quantum dot behavior in tumors, Biochim. Biophys. Acta-Gen. Subj., 1862 (2018) 914-923.

[30] M. Vinci, S. Gowan, F. Boxall, L. Patterson, M. Zimmermann, W. Court, C. Lomas, M. Mendiola, D. Hardisson, S.A. Eccles, Advances in establishment and analysis of three-dimensional tumor spheroid-based functional assays for target validation and drug evaluation, BMC Biol., 10 (2012). 
[31] B. Hegedus, F. Marga, K. Jakab, K.L. Sharpe-Timms, G. Forgacs, The interplay of cell-cell and cell-matrix interactions in the invasive properties of brain tumors, Biophys. J., 91 (2006) 2708-2716.

[32] M.K. Ediriweera, K.H. Tennekoon, S.R. Samarakoon, In vitro assays and techniques utilized in anticancer drug discovery, J. Appl. Toxicol., 39 (2019) 38-71.

[33] A. Ivascu, M. Kubbies, Rapid generation of single-tumor spheroids for high-throughput cell function and toxicity analysis, J. Biomol. Screen., 11 (2006) 922-932.

[34] E.B. Berens, J.M. Holy, A.T. Riegel, A. Wellstein, A cancer cell spheroid assay to assess invasion in a 3D setting, J. Vis. Exp., 2015 (2015) e53409.

[35] K.M. Yamada, E. Cukierman, Modeling tissue morphogenesis and cancer in 3D, Cell, 130 (2007) 601-610.

[36] Z.Y. Liu, L. Speroni, K.P. Quinn, C. Alonzo, D. Pouli, Y. Zhang, E. Stuntz, C. Sonnenschein, A.M. Soto, I. Georgakoudi, 3D organizational mapping of collagen fibers elucidates matrix remodeling in a hormone-sensitive 3D breast tissue model, Biomaterials, 179 (2018) 96-108.

[37] N. Ohtsu, A threshold selection method from gray-level histograms, IEEE Trans. Syst. Man Cybern. -Syst., 9 (1979) $62-66$.

[38] A.M. Stein, T. Demuth, D. Mobley, M. Berens, L.M. Sander, A mathematical model of glioblastoma tumor spheroid invasion in a three-dimensional in vitro experiment, Biophys. J., 92 (2007) 356-365.

[39] J. Friedrich, C. Seidel, R. Ebner, L.A. Kunz-Schughart, Spheroid-based drug screen: considerations and practical approach, Nat Protoc, 4 (2009) 309-324.

[40] M.A. Solomon, J. Lemera, G.G.M. D'Souza, Development of an in vitro tumor spheroid culture model amenable to high-throughput testing of potential anticancer nanotherapeutics, J. Liposome Res., 26 (2016) 246-260.

[41] O.D. Wever, H. An, A.D. Boeck, W. Westbroek, G. Braems, S. Emami, M. Sabbah, C. Gespach, M. Bracke, Modeling and quantification of cancer cell invasion through collagen type I matrices, Int. J. Dev. Biol., 54 (2009) 887-896.

[42] Y. Hou, J. Konen, D.J. Brat, A.I. Marcus, L. Cooper, TASI: A software tool for spatial-temporal quantification of tumor spheroid dynamics, Sci Rep, 8 (2018) 7248.

[43] D.D. Chen, L. Yang, X.J. Chen, X.H. Zhang, Y.M. Liu, Z.Q. Guo, L.W. Zhang, Automated contour analysis of multi-cellular spheroids spreading through high content imaging, Phys Biol, 15 (2018).

[44] H. Huang, R.A. Svoboda, A.J. Lazenby, J. Saowapa, N. Chaika, K. Ding, M.J. Wheelock, K.R. Johnson, Up-regulation of $\mathrm{N}$-cadherin by Collagen I-activated discoidin domain receptor 1 in pancreatic cancer requires the adaptor molecule Shc1, J. Biol. Chem., 291 (2017) 23208-23223.

[45] H. Jin, I.H. Ham, H.J. Oh, C.A. Bae, D.K. Lee, Y.B. Kim, S.Y. Son, Y.J. Chwae, S.U. Han, R.A. Brekken, Inhibition 
of discoidin domain receptor 1 prevents stroma-induced peritoneal metastasis in gastric carcinoma, Mol. Cancer Res., 16 (2018) 1590 .

[46] H.C. Cristina, H. Steven, S.I. Chaudhry, W. Peter, H. Kevin, L. Birgit, S. Erik, Collective cell migration requires suppression of actomyosin at cell-cell contacts mediated by DDR1 and the cell polarity regulators Par3 and Par6, Nat. Cell Biol., 13 (2011) 49-58.

[47] Q. Shen, V.R. Cicinnati, X. Zhang, S. Iacob, F. Weber, G.C. Sotiropoulos, A. Radtke, M. Lu, A. Paul, G. Gerken, Role of microRNA-199a-5p and discoidin domain receptor 1 in human hepatocellular carcinoma invasion, Mol. Cancer, 9 (2010) 227.

[48] C. Ambrogio, G. Gomez-Lopez, M. Falcone, A. Vidal, E. Nadal, N. Crosetto, R.B. Blasco, P.J. Fernandez-Marcos, M. Sanchez-Cespedes, X.M. Ren, Z. Wang, K. Ding, M. Hidalgo, M. Serrano, A. Villanueva, D. Santamaria, M. Barbacid, Combined inhibition of DDR1 and Notch signaling is a therapeutic strategy for KRAS-driven lung adenocarcinoma, Nat. Med., 22 (2016) 270-277.

[49] Q.P. Lu, W.D. Chen, J.R. Peng, Y.D. Xu, Q. Cai, G.K. Feng, K. Ding, X.F. Zhu, Z. Guan, Antitumor activity of 7RH,

a discoidin domain receptor 1 inhibitor, alone or in combination with dasatinib exhibits antitumor effects in nasopharyngeal carcinoma cells, Oncol Lett, 12 (2016) 3598-3608.

[50] M.S. Gao, L. Duan, J.F. Luo, L.W. Zhang, X.Y. Lu, Y. Zhang, Z. Zhang, Z.C. Tu, Y. Xu, X.M. Ren, K. Ding, Discovery and Optimization of 3-(2-(Pyrazolo[1,5-a]pyrimidin-6-yl)-ethynyl)benzamides as Novel Selective and Orally Bioavailable Discoidin Domain Receptor 1 (DDR1) Inhibitors, J. Med. Chem., 56 (2013) 3281-3295. 


\section{Figure captions}

\section{Table 1}

Statistic results of PTI transition time evaluated from various parameters. Also see the brief summary in Fig. 4(c).

Fig. 1. Schematic diagram of the home-built system for identification of proliferation-to-invasion (PTI) transition in the in vitro tumor spheroid model. (a) The transition from tumor-cell proliferation to invasion is critical, but is still insufficient in research. (b) Cells at a density of $1.0 \times 10^{4}$ cells $/ \mathrm{mL}$ were seeded in the 96-well round bottom ultra-low attachment (ULA) plate where, after 96 hours, spheroids formed. The spheroids were then transferred to a culture medium with an additional $2.0 \mathrm{mg} / \mathrm{mL}$ collagen I in another 96-well flat bottom ULA plate. This allows the PTI transition to take place. (c-d) Workflow of the home-built living-cell incubation system for inverted optical microscopes and photos of the corresponding components. The control system (c) consists of a $\mathrm{CO}_{2}$ controller and several temperature controllers. The incubator holder contains a humidifier module, four temperature sensors and heaters, and supports a 96-well plate or a $60 \mathrm{~mm}$ petri dish.

Fig. 2. Flowchart of the spheroid boundary analysis. (a) Work flow of image processing. The original fluorescence image was gray scaled, then smoothed to remove background noises, and finally spheroid was identified as the largest connected component in the center. Scale bar, $200 \mu \mathrm{m}$. (b) The boundaries of the identified spheroid (U87 cells) at 0, 40, 80, and 120 min.

Fig. 3. Boundary analysis procedure. The result from a U87 spheroid is presented as a demonstration. The original spheroid boundary (a1) at $50 \mathrm{~min}$ in the polar coordinate is divided into 300 sections. The boundary data in each radian section (a2) is utilized to define the corresponding edge value by the furthest point to the center (a3); consequently, this process results in a smoothed boundary (a4) for further analysis. (b) The discrete Fourier transform of boundary into the frequency domain, where the high-frequency component (> 15) is chosen (blue) for the sharp protrusion boundary, resulted from collective cell invasion (see SI Fig. S4 for details of choosing the separation frequency). (c) The distance of boundary from the center vs. radian: smoothed boundary $\left(d\right.$, red), the high frequency component $\left(d_{h f}\right.$, blue), the low frequency component $\left(d_{l f}\right.$, purple), and the average value ( $d_{\text {avg }}$, cyan) from the low-frequency component. (d) The trajectories of perimeter ${ }^{2}$-to-area ratio $\left(r_{p t a}\right)$ and the standard deviation of relative boundary-to-center distance $d_{s t d}$ of this U87 spheroid vs. tracking time. The PTI transition time is identified to be 40 min from both trajectories.

Fig. 4. Variations in the protrusion height $(h)$ and height-to-width ratio $\left(r_{h t w}\right)$ of U87 spheroids, and the results for transition time $\left(\tau_{p t i}\right)$ of three cell lines. (a) The probability distribution of $h$ and $r_{h t w}$ measured for U87 spheroids. Here, the large dense domain at the bottom-left corner is attributed to protrusions with small $h$ and low $r_{h t w}$ from the proliferation dynamics of spheroids. The probability domains in the upper-right portion of the histogram (yellow square, $h>18 \mu \mathrm{m}$ and $r_{h t w}>0.55$ ) are rare events and are used to identify invasion 
protrusions. The red dashed and dash-dot lines indicate the thresholds for single-parameter justifications, i.e. $h>20 \mu \mathrm{m}$ or $r_{h t w}>0.6$. (b) The occurrence of protrusions identified by either double or single parameters from (a) vs. the imaging time. The dashed lines indicate the identified transition points: 40 min from double-parameter justification and from $r_{h t w}>0.6$, and $50 \mathrm{~min}$ from $h>20 \mu \mathrm{m}$. (c) The average $\tau_{p t i}$ obtained from spheroids ( $n=3$ ) of U87 cells, H1299 cells and MDA-MB-231 cells without (gray column) and with (black column) DDR1 inhibitor 7rh, and H1299 cells of bright-field images (white column). Data are presented as means $\pm \mathrm{SD}$ of three independent samples. Values of $\tau_{p t i}$ were $47 \pm 5 \mathrm{~min}$ (without $7 \mathrm{rh}$ ) and $61 \pm$ $2 \mathrm{~min}$ (with 7rh) for U87 cells, $61 \pm 16 \mathrm{~min}$ (without 7rh) and $97 \pm 10 \mathrm{~min}$ (with 7rh) for MDA-MB231 cells. For H1299 cells. Values of $\tau_{p t i}$ were $64 \pm 14$ min (fluorescence, without 7rh) and $89 \pm 3$ min (fluorescence, with $7 \mathrm{rh}$ ), and $69 \pm 10 \mathrm{~min}$ for bright-field images corresponding to fluorescence images without $7 \mathrm{rh}$. $* P<$ 0.05 . 


\section{Conflict of Interest form statement:}

We have no conflict of Interest. 
Highlights

- Proliferation-to-invasion transition model by tumor spheroids in defined conditions

- Image-based, automated and quantitative analysis of the morphological transitions

- Proposed suitable parameters for well definition of the transition point

- Transition point and time provide good assistance for cancer invasion investigation 


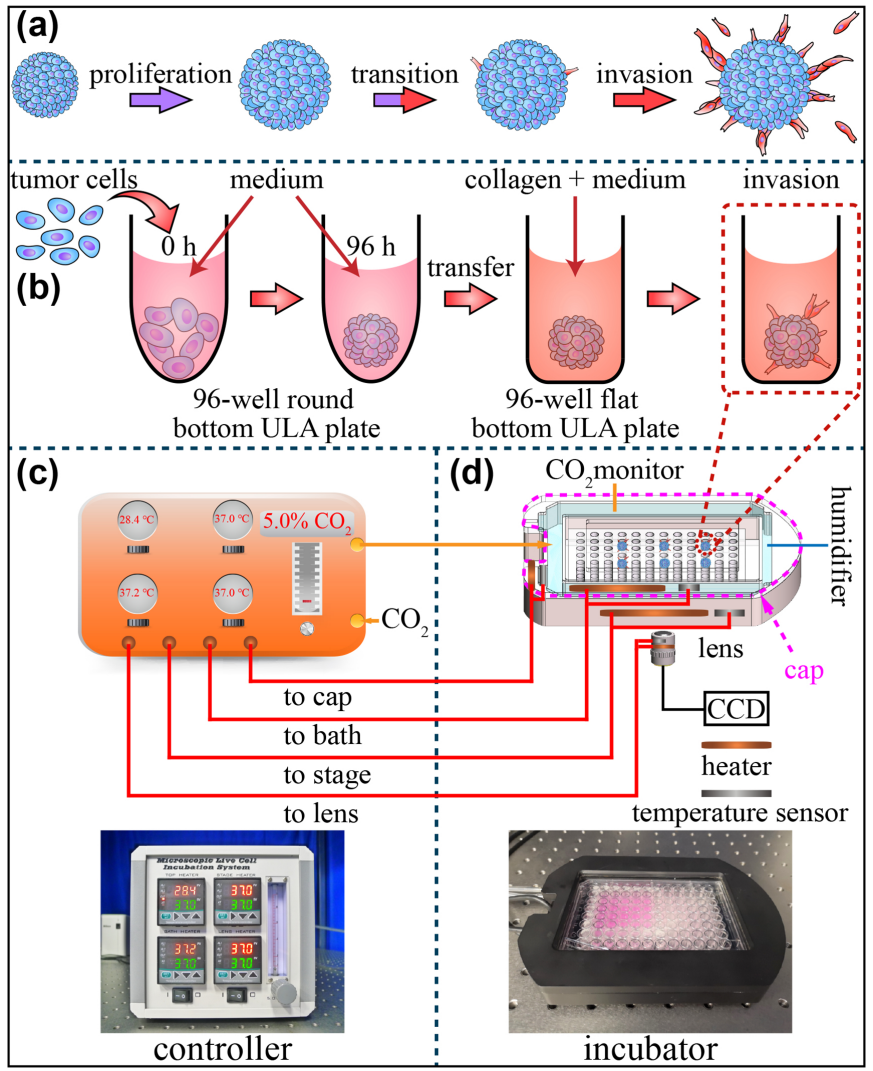

Figure 1 


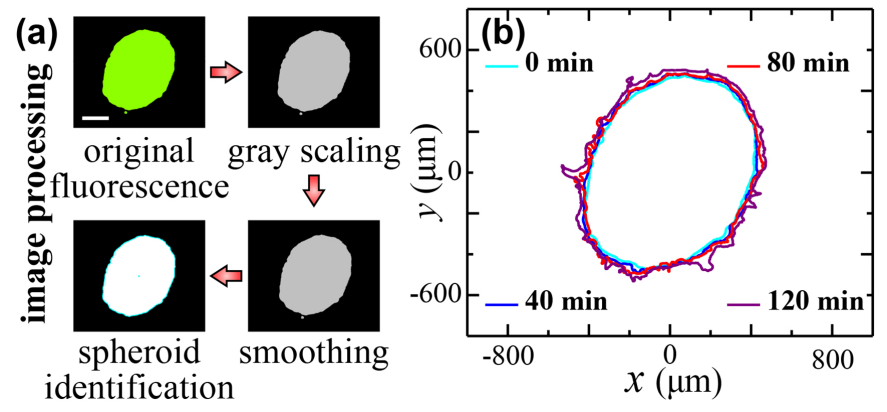

Figure 2 


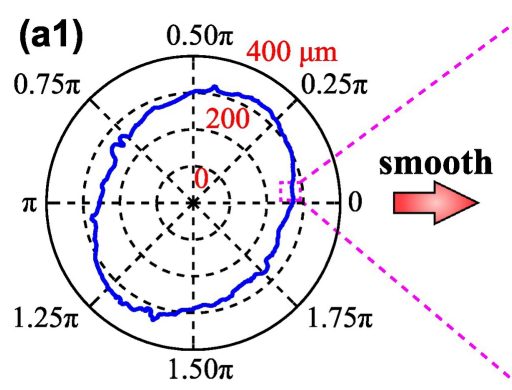

original boundary

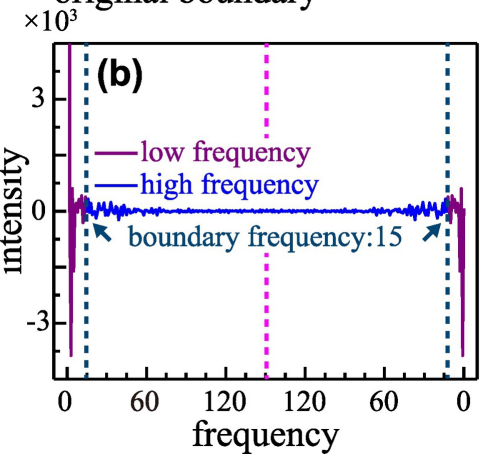

\section{(a2)} ह 긍

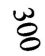

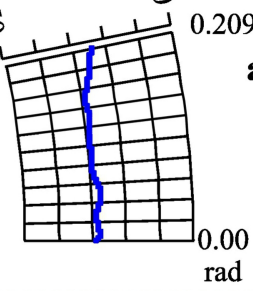

boundary smoothing

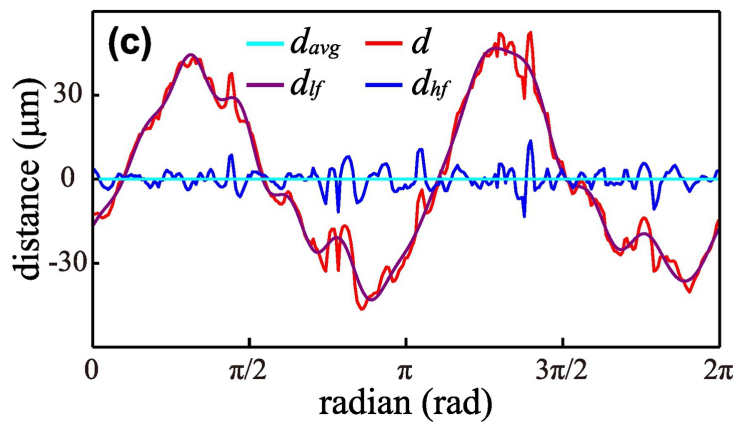

(a4) 0.209

\section{average}

हू

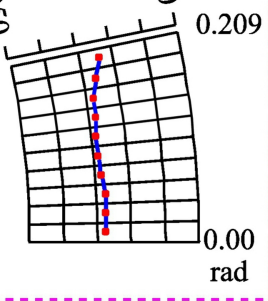

$\mathrm{ad}$

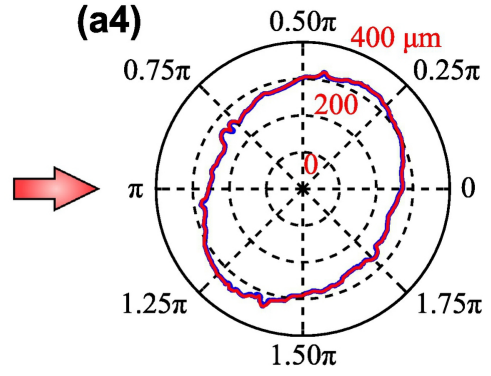

smoothed boundary $\times 10^{-2}$

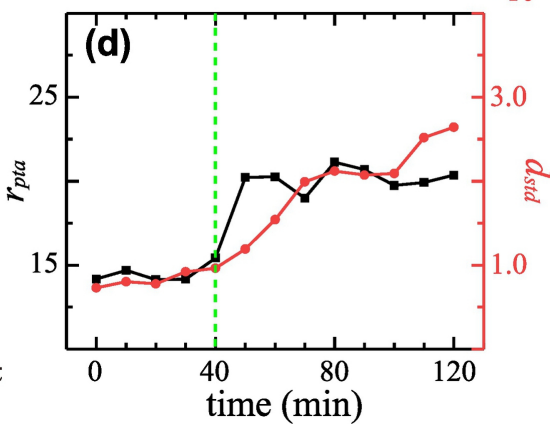

Figure 3 
(a)

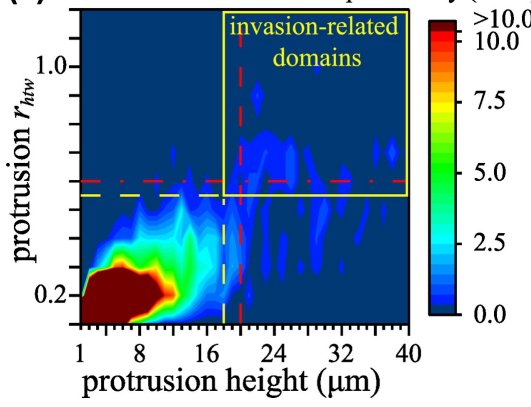

(b)

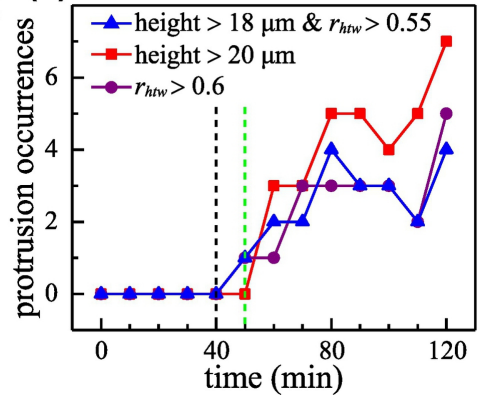

(c)

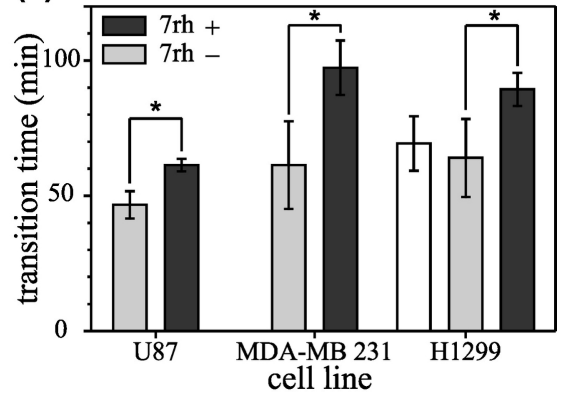

Figure 4 